\title{
Thermo-Optical Study of Cholesteric Liquid Crystal and Mixtures
}

\author{
Radha Srinivasan $^{1}$, Dr. Rita Gharde, Bhave Manisha ${ }^{3}$ \\ ${ }^{1}$ Department of Physics, University of Mumbai, Santacruz (E), Mumbai-98, India \\ ${ }^{2}$ Siddharth College of Arts, Science and Commerce, Fort, Mumbai-1, India
}

\begin{abstract}
In this paper an experimental Thermal study of Cholesteric Liquid Crystal and it's mixtures is presented. We have dispersed monomer in CLC to form Polymer dispersed liquid crystal. We have also doped ferroelectric nano - powder of Barium titanate (BaTiO3) in CLC. Different analytical techniques were used to study the phase morphology of CLC in the neat as well as in the doped state. Differential scanning calorimetry and Fabry-Perot spectroscopy techniques were employed to study phase transitions. The mesophase characterization was done using optical polarizing microscopy. The CLC, both as mesogenic moieties and as dispersed droplets exhibited various textures according to their molecular order and orientation. The phase transitions occurred at temperatures lower than those exhibited by the mesogenic component before doping. Enhancement in the thermo-optical properties of CLC is observed after doping. This holds potential for variety of electro-optic application ranging from display to light shutters.
\end{abstract}

Keywords: Cholesteric liquid crystal (CLC), Optical polarising microscopy (PMS), Fabry-perot spectroscopy (FPSS), Differential scanning calorimetry (DSC), Phase transition temperature (PTT).

\section{Introduction}

Liquid crystals are self-organizing anisotropic soft materials which are widely used in electro-optical applications. Particularly interesting are LC composites based on chiral nematic liquid crystals (cholesterics). These materials exhibit selective reflection and giant optical activity that can easily be regulated by electric field and temperature. Polymer Dispersed Liquid Crystals (PDLC) is formed by phase separation of the LC component from a homogeneous solution with a monomer or polymer [1]. The liquid crystal forms droplets whose size, shape and distribution depend on the technique used. These tiny droplets (a few microns across for practical applications) are responsible for the unique behavior of the material. PDLC films have a remarkable electro-optical behavior since they can be switched from highly light scattering state (OFF) to transparent state (ON) simply by application of an electric field $[2,3,4,5]$. PDLC films can be prepared by four different techniques namely Microencapsulation, Thermally Induced Phase Separation (TIPS), Solvent Induced Phase Separation (SIPS) and Polymerization Induced Phase Separation (PIPS) [6, 7, and 8]. Related work shows that the liquid crystal doped with polymer as well as monomer enhances physical as well as optical properties of the material which improves the thermal and mechanical stability of thermooptical devices $[9,10]$. Recently, nanoparticals have been doped into Liquid Crystals and studied for improvement in electro-optic and optical properties such as enhanced photoluminescence, higher polarization, fast response time, low operating voltage, and improved conductivity [11-20]. For enhancing the physical properties a proper selection of nonmaterial for liquid crystals is required. This depends upon various factors such as size, shape, preparation methods, surfactant concentration, and amount of doping materials $[21,22]$. In the present study our main focus is on the characterization of mesophases before and after doping CLC.

\section{List of Chemicals and sample preparation}

The CLC in the present study, Cholesteryl Myristate is a thermotropic liquid crystal whose properties change with the change in temperature.

CLC was dispersed with monomer to form polymer dispersed liquid crystal. The monomer Ethylene Glycol Dimethacrylate (EGDMA) is a water-insoluble diffunctional methacrylic monomer employed as a cross-linking agent or a low viscosity reactive diluent. EGDMA was dispersed in CLC by encapsulation method. The PDLC formed contained $10 \%$ of EGDMA with $90 \%$ of CLC.

We have used ferroelectric nano - powder of Barium titanate (BaTiO3) to disperse in the CLC. The $\mathrm{BaTiO} 3$ particles are slightly anisotropic and their size is less than $100 \mathrm{~nm}$. BaTiO3 were mixed with Oleic acid and Heptane in appropriate proportion by weight and then this mixture was doped with CLC by ultra-sonication method. The ultra-sonicaton ensures homogeneous distribution of nano - powder in Cholesteric liquid crystal. The mixture was kept in vacuum for six hours for complete evaporation of heptane. The resulting sample contains the small concentration $(\sim 1 \%)$ of $\mathrm{BaTiO} 3$ nano particles

Samples:

CLC: Cholesteryl Myristate Molecular formula: $\mathrm{C}_{41} \mathrm{H}_{72} \mathrm{O}_{2}$, Melting Point: $84^{\circ} \mathrm{C}$

Mixtures:

CLC+M: Cholesteryl Myristate +EGDMA

CLC+N: Cholesteryl Myristate $+\mathrm{BaTiO} 3$

\section{Experimental Techniques}

The essential tools used in present study are Differential scanning calorimetry (DSC), Fabry-perot spectroscopy (FPSS) and Polarizing microscopy (PMS). We have used a 


\section{International Journal of Science and Research (IJSR) \\ ISSN (Online): 2319-7064 \\ Index Copernicus Value (2013): 6.14 | Impact Factor (2015): 6.391}

combination of detection techniques, including both optical and thermal measurements in order to ensure the characterization of the sample as complete as possible.

\section{1) Differential scanning calorimetry (DSC):}

DSC is a thermoanlytical technique in which the difference in the amount of heat required to increase the temperature of the sample and reference is measured as a function of temperature. Both the sample and reference are maintained nearly at the same temperature throughout the experiment. The temperature of the sample holder increases linearly as a function of time. Even small changes in thermodynamic quantities at transitions between mesophases can be detected by DSC. The result of a DSC experiment is a curve of heat flux versus temperature or versus time. Most liquid crystal to liquid crystal transitions are discontinuous. Such transitions are revealed by larger peaks.

\section{2) Fabry-perot spectroscopy (FPSS):}

In FPSS, sample scatters the incident laser light at 90 degrees to the incident beam. This scattered light was allowed to fall on the Fabry-Perot Etalon and the Fabry-Perot rings were observed through the spectrometer eye-piece. The diameters of one to five rings were measured at various temperatures. At the clearing point the ring structure becomes blurred and no readings can be taken thereafter. Similar measurements were carried out while cooling the isotropic liquid to liquid crystal. Sufficient number of heating cycles and cooling cycles were performed for pure and doped liquid crystal so as to determine phase transition temperatures.

\section{3) Polarizing Microscopy (PMS):}

Depending upon the local orientation of the molecules, a thin layer of the sample sandwiched between the two glass plates when observed under cross polarisers, give rise to beautiful patterns called textures. The textures are unique to the particular type of the liquid crystal phase. Polarizing Microscopy technique enables the identification of the type of liquid crystal and other mesophases from the optical texture that is generated. We obtained the PMS results using a polarizing microscope (Carl Zeiss Jena).

\section{Results and Discussion}

\section{A. Differential scanning calorimetry (DSC)}

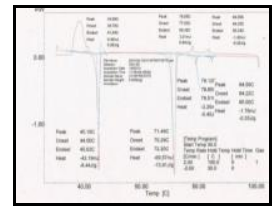

Fig.1.a

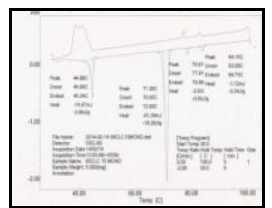

Fig.1.b

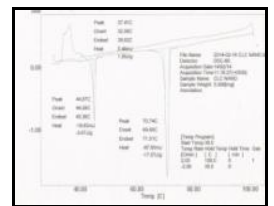

Fig.1.c
Figure 1: DSC thermograph of a) CLC b) CLC $+\mathrm{M}$ c) $\mathrm{CLC}+\mathrm{N}$

The figures 1.a, 1.b and 1.c show the DSC thermographs for pure $\mathrm{CLC}, \mathrm{CLC}+\mathrm{M}$ and $\mathrm{CLC}+\mathrm{N}$ respectively.
Table 1: PTTS observed by DSC

\begin{tabular}{|c|c|c|c|c|}
\hline Sample & \multicolumn{2}{|c|}{ Heating } & \multicolumn{2}{c|}{ Cooling } \\
\hline & $\mathrm{PTT}^{0} \mathrm{C}$ & $\Delta \mathrm{H} \mathrm{J} / \mathrm{g}$ & $\mathrm{PTT}^{0} \mathrm{C}$ & $\Delta \mathrm{H} \mathrm{J} / \mathrm{g}$ \\
\hline CLC & 45.19 & -8.44 & & \\
\hline & 71.49 & -13.91 & 34.09 & 0.08 \\
\hline & 79.12 & -0.45 & 76.05 & 0.64 \\
\hline & 84.59 & -0.35 & 84.59 & -0.28 \\
\hline CLC + M & 44.88 & -3.99 & & \\
\hline & 71.38 & -18.28 & 44.88 & -0.37 \\
\hline & 78.61 & -0.59 & 77.21 & 0.40 \\
\hline & 84.15 & -0.34 & 83.01 & 0.28 \\
\hline CLC + N & 44.97 & -3.97 & & \\
\hline & 70.4 & -17.57 & 37.41 & 1.09 \\
\hline
\end{tabular}

The PTTs for CLC, CLC+M, CLC+N are presented in table 1.Though the significant change in the PTTs of CLC are not observed after addition of the monomer, the doping of ferroelectric nanopowder reduces PTTs by $14^{0} \mathrm{C}$.

\section{B. Fabry-perot spectroscopy (FPSS):}

The graphical mappings of diameter of Fabry-Perot rings vs. temperature in the Fabry-perot spectroscopy gives us the information about transition temperatures of the mesophases. The graph of diameter of Fabry-Perot rings vs. temperature shows an abrupt variation. (20' to $25^{\prime}$ 'with spectrometer of least count $=30$ ') at the mesophase transition temperatures as shown in Figures 2, 3 and 4.
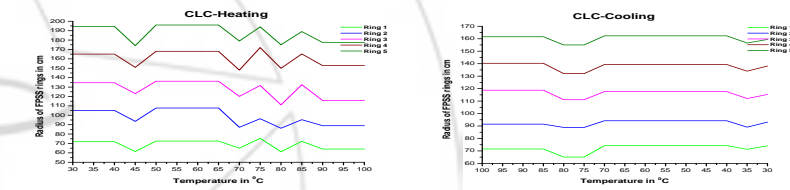

Figure 2: Phase Diagrams for CLC
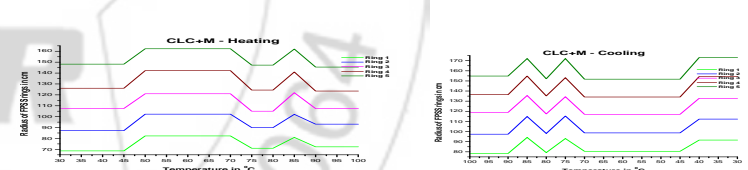

Figure 3: Phase Diagrams for CLC $+M$
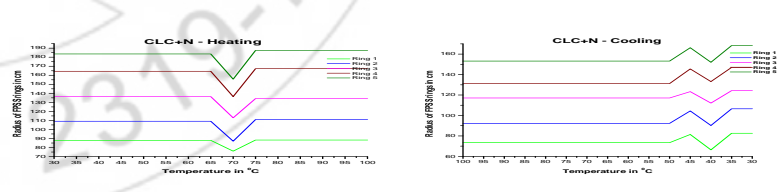

Figure 4: Phase Diagrams for CLC + NP

\section{Optical Polarising Microscopy:}

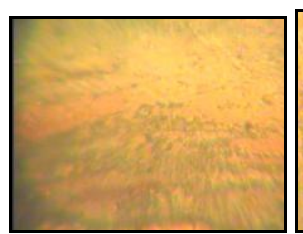

Fig.5.a

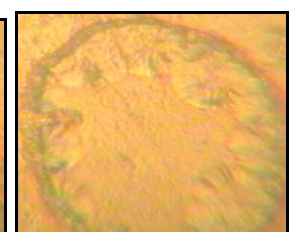

Fig.5.b

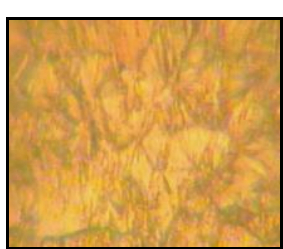

Fig.5.c
Figure 5: Textures of CLC

a) Smectic Phase at $77.6^{\circ} \mathrm{C}$ cooling

b) Appearance of Fan shaped cholesteric texture at $\mathbf{8 0 . 8}^{0} \mathrm{C}$ while heating

c) Fan shaped cholesteric texture at $83.9^{\circ} \mathrm{Cwhile}$ heating. 


\section{International Journal of Science and Research (IJSR) \\ ISSN (Online): 2319-7064}

Index Copernicus Value (2013): 6.14 | Impact Factor (2015): 6.391

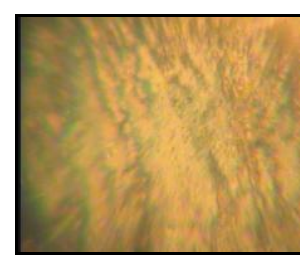

Fig.6.a

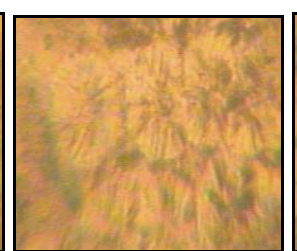

Fig.6.b

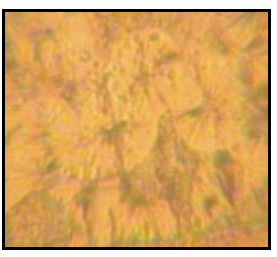

Fig.6.c
Figure 6: Textures of CLC $+\mathrm{M}$

a) Smectic Phase at $74.7^{\circ} \mathrm{C}$ cooling

b) Appearance of cholesteric Fan shaped texture at $77.2^{0} \mathrm{C}$ while heating

c) Cholesteric Fan shaped texture at $79.2^{\circ} \mathrm{C}$ while heating.

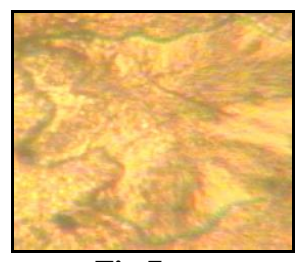

Fig.7.a

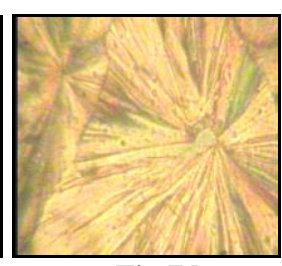

Fig.7.b

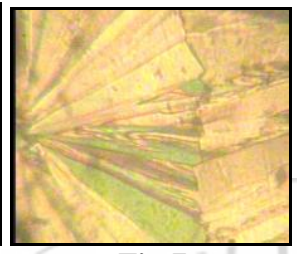

Fig.7.c
Figure 7: Textures of $\mathrm{CLC}+\mathrm{N}$

a) Smectic Phase at $56.7^{0} \mathrm{C}$ while heating

b) Appearance of cholesteric Fan shaped texture appeared at $56.9^{\circ} \mathrm{C}$ while cooling

c) Cholesteric Fan shaped texture at $54.4^{\circ} \mathrm{C}$ while cooling.

The textures observed for CLC, CLC $+\mathrm{M}$ and $\mathrm{CLC}+\mathrm{N}$ are presented in Figure 5, 6 and 7 respectively. The textures appear as per the phase and the temperature sequence pertaining to the sample.

Table 2: PTTS observed by DSC, FPSS and PMS

\begin{tabular}{|c|c|c|c|}
\hline Sample $\rightarrow$ & \multirow{2}{*}{$\begin{array}{c}\text { CLC } \\
\left(\mathrm{PTT}^{0} \mathrm{C}\right)\end{array}$} & \multirow{2}{*}{$\begin{array}{l}\mathrm{CLC}+\mathrm{M} \\
\left(\mathrm{PTT}^{0} \mathrm{C}\right)\end{array}$} & \multirow{2}{*}{$\begin{array}{l}\mathrm{CLC}+\mathrm{N} \\
\left(\mathrm{PTT}^{0} \mathrm{C}\right)\end{array}$} \\
\hline $\begin{array}{c}\text { Technique } \\
\downarrow\end{array}$ & & & \\
\hline DSC & $\begin{array}{l}34.09,45.19 \\
71.49,76.05 \\
79.12,84.59\end{array}$ & $\begin{array}{l}44.88,71.38, \\
77.21,78.61, \\
83.01,84.15\end{array}$ & $\begin{array}{c}37.41,44.97 \\
70.74\end{array}$ \\
\hline FPSS & $\begin{array}{l}35,40,45,65 \\
70,75,80,85\end{array}$ & $\begin{array}{c}45,70,80,85 \\
90\end{array}$ & $\begin{array}{c}40,45,50 \\
65,70\end{array}$ \\
\hline PMS & $77.6,80.8,83.9$ & $\begin{array}{c}74.7,77.2, \\
79.2 \\
\end{array}$ & $\begin{array}{c}54.4,56.7 \\
56.9 \\
\end{array}$ \\
\hline
\end{tabular}

The PTTS observed by all the three techniques are presented in Table 2.The PTTs obtained by DSC are confirmed by FPSS. The appearance of textures at some of these temperatures confirms the values of PTTs further.

\section{Conclusion}

The mesomorphic behavior of pure Cholesteric Liquid crystal, Polymer dispersed liquid crystal and Cholesteric liquid crystal dispersed with ferroelectric nanopowder at a low concentration was studied using DSC, FPSS, and PMS. Due to spatial confinement and an interphase of adsorbed mesogen at the droplet boundaries, the mesophase transition for PDLC occurred at a lower temperature than CLC in pure state. The textures similar to the textures of pure CLC were observed for doped CLC at lower temperatures. The low concentration of $\mathrm{BaTiO} 3$ nanoparticles in CLC host increases the orientational ordering of the liquid crystal resulting in lower phase transition temperatures. These modified properties of the liquid Crystals are caused by the interaction of the nanoparticles with the liquid Crystals. This enhancement in thermooptical property holds promise in variety of thermooptical applications.

\section{Acknowledgement}

I would like to dedicate this paper to Late Dr.Rita Gharde under whom I started work on this paper. I also thank Dr. Radha Srinivasan for guiding me to complete this paper. I would like to thank Dr.Anuradha Misra, Head, Department of Physics, University of Mumbai and Dr.G.V.Rao, I/C Principal, Siddharth College for supporting my work.

\section{References}

[1] R. A. WEISS and C. K. OBER, "ACS Symposium Series," Vol. 435 (1990) Ch. 32, pp. 475-495.

[2] Drazic, P. S. (1995). Liquid Crystal Dispersion, World Scientific, Singapore.

[3] Doane, J. W., Golemme, A., West, J. L., Whitehead, J. B., \& Wu, B. G. (1988).Mol. Cryst. Liq. Cryst. 165, 511.

[4] Smith, G. W. (1993). Mol. Cryst. Liq. Cryst., 225, 113.

[5] Simoni, F. \& Francescangeli, O. (2000). Intern. J. Polymeric Mater., 45, 381.

[6] C.W. Smith Mol. Cryst. Liq. Cryst., 225 (1993), p. 113

[7] C.W. Smith Int. J. Mod. Phys. B, 7 (1993), p. 4187

[8] S.C. Jain, D.K. Rout J. Appl. Phys., 70 (1991), p. 6988

[9] Heynderickx and D. Broer, "Thermo-Optical Properties of a Polymer Dispersed Liquid Crystalline Polymer," Molecular Crystals and Liquid Crystals Science and Technology, vol. 263, pp. 415-427, 1995.

[10]D. Yang, J. Lin, T. Li, S. Lin, and X. Tian, "Effect of external electrical field on phase behavior and morphology development of polymer dispersed liquid crystal," European Polymer Journal, vol. 40, p. 1823, 2004.

[11] J. Prakash, A. Choudhary, A. Kumar, D. S. Mehta, and A. M. Biradar, "Nonvolatile memory effect based on gold nanoparticles doped ferroelectric liquid crystal," Applied Physics Letters, vol. 93, no. 11, Article ID 112904, 2008.

[12]Kumar, J. Prakash, D. S. Mehta, W. Haase, and A. M. Biradar, "Enhanced photoluminescence in gold nanoparticles doped ferroelectric liquid crystals," Applied Physics Letters, vol. 95, no. 2, Article ID 023117, 2009.

[13]X. Tong and Y. Zhao, "Liquid-crystal gel-dispersed quantum dots: reversible modulation of photoluminescence intensity using an electric field," Journal of the American Chemical Society, vol. 129, no. 20, pp. 6372-6373, 2007.

[14] S. W. Lee, C. Mao, C. E. Flynn, and A. M. Belcher, "Ordering of quantum dots, using genetically engineered viruses," Science, vol. 296, no. 5569, pp. 892-895, 2002.

[15]Balandin, K. L. Wang, N. Kouklin, and S. Bandyopadhyay, "Raman spectroscopy of 
electrochemically self-assembled $\mathrm{CdS}$ quantum dots," Applied Physics Letters, vol. 76, no. 2, pp. 137$139,2000$.

[16]H. L. Lee, I. A. Mohammed, M. Belmahi, M. B. Assouar, H. Rinnert, and M. Alnot, "Thermal and optical properties of $\mathrm{CdS}$ nanoparticles in thermotropic liquid crystal monomers," Materials, vol. 3, pp. 2069-2086, 2010.

[17] S. Pandey, R. Dhar, S. Kumar, and R. Dabrowski, "Enhancement of the display parameters of 4'-pentyl-4cyanobiphenyl due to the dispersion of functionalised gold nano particles," Liquid Crystals, vol. 38, no. 1, pp. 115-120, 2011.

[18]F. V. Podgornov, A. M. Suvorova, A. V. Lapanik, and W. Haase, "Electrooptic and dielectric properties of ferroelectric liquid crystal/single walled carbon nanotubes dispersions confined in thin cells,"Chemical Physics Letters, vol. 479, no. 4-6, pp. 206-210, 2009.

[19] Chaudhary, P. Malik, R. Mehra, and K. K. Raina, "Electro-optic and dielectric studies of silica doped ferroelectric liquid crystal in $\mathrm{SmC} *$ phase," Phase Transitions, vol. 85, pp. 244-254, 2012.

[20]P. Malik, A. Chaudhary, R. Mehra, and K. K. Raina, "Electro-optic, thermo-optic and dielectric response of multiwalled carbon nanotube doped ferroelectric liquid crystal thin films," Journal of Molecular Liquids, vol. 165, pp. 7-11, 2012.

[21] Kumar, J. Prakash, M. T. Khan, S. K. Dhawan, and A. M. Biradar, "Memory effect in cadmium telluride quantum dots doped ferroelectric liquid crystals," Applied Physics Letters, vol. 97, no. 16, Article ID 163113, 3 pages, 2010.

[22] P. Malik, A. Chaudhary, R. Mehra, and K. K. Raina, "Dielectric studies and memory effect in nanoparticle doped ferroelectric liquid crystal films," Molecular Crystals and Liquid Crystals, vol. 541, pp. 243-251, 2011. 